\title{
Zika virus Pathogenesis in Infant Mice after Natural Transmission by the Bite of Infected Mosquitoes
}

\author{
Pragya D. Yadav ${ }^{a}$ Vimal Kumar ${ }^{b}$ Sandeep Kumara Chandrashekhar S. Mote ${ }^{c}$ \\ Triparna D. Majumdar ${ }^{a}$ Mangesh Gokhale ${ }^{a}$ Pravin Kore $^{a}$ \\ Devendra T. Mourya ${ }^{a}$
}

${ }^{\mathrm{a}}$ Microbial Containment Complex, National Institute of Virology, Pune, India; ${ }^{\mathrm{b}}$ National Jalma Institute of Leprosy and Other Mycobacterial Diseases, Agra, India; ' Krantisinh Nana Patil College of Veterinary Sciences, Satara, India

\section{Keywords}

Zika virus · Pathogenesis · CD1 mice ·

Immunohistochemistry · Histopathology

\section{Abstract}

Objectives: The objective of this study was to understand natural disease progression in infant CD1 mice after the bite of Aedes aegypti mosquitoes infected by the Zika virus (ZIKV, MR-766 strain). Methods: A. aegypti mosquitoes were experimentally infected with ZIKV MR-766 strain via the oral feeding route. Infected mosquitoes were allowed to feed on infant CD1 mice. Sick mice were euthanized, and their organs were collected and subjected to real-time RT-PCR, histopathology, and immunohistochemistry. Results: Clinical symptoms appeared in mice after 4-5 days of being bitten by mosquitoes, following which they were euthanized. Real-time RT-PCR analysis showed the presence of viral RNA in various organs such as the brain, liver, kidney, spleen, lungs, and intestines of the mice. The brain tissue specimens showed higher viral loads as determined by threshold values (Ct value) in the real-time RT-PCR assay. Histopatholog- ical and immunohistochemistry studies also revealed the presence of the virus and associated lesions in the brain, indicating that ZIKV shows tropism for neuronal tissue. Conclusions: This study demonstrates ZIKV pathogenesis in infant CD1 mice and that these mice are highly susceptible to natural infection with this ZIKV strain.

(c) 2018 S. Karger AG, Base

\section{Introduction}

Zika virus (ZIKV) belongs to the Flaviviridae family, genus Flavivirus of single-stranded RNA viruses. Since its isolation in the Zika Forest of Uganda in 1947 [1,2], it had been observed that the virus was restricted to Africa and Asia, with rare confirmed cases showing mild symptoms, such as rash, fever, headaches, arthralgia, and conjunctivitis [3]. Recently, in 2015, there was a sudden spurt in cases of ZIKV in Brazil, which came as a surprise, as it had not been previously reported in this part of the world [4]. Now, with its presence in at least 24 countries and territories, ZIKV has emerged as a public health emergency.
๑) 2018 S. Karger AG, Basel
Dr. D.T. Mourya

Microbial Containment Complex, National Institute of Virology

20-A, Dr. Ambedkar Road

Pune, Maharashtra 411001 (India)

E-Mail directorniv@gmail.com,dtmourya@gmail.com 
The virus is believed to have originated in Africa, where it circulated enzootically in nonhuman primates and was transmitted by arboreal Aedes mosquitoes [5]. Human cases resulted from a human-mosquito cycle involving Aedes aegypti [6], which is probably the major reason for its spread to other areas of the globe, including most tropical and subtropical regions such as South-East Asia Region (SEAR) countries. High population densities accompanied by the huge presence of vectors in these areas are highly conducive to the spread of ZIKV [7].

Signs and symptoms of ZIKV infection resemble that of other arboviral diseases including dengue fever [8]. Moreover, the ZIKV infection has been observed to be associated with increasing cases of microcephaly, Guillain-Barré syndrome, and other neurological abnormalities and congenital malformations [9]. Based on Bradford Hill viewpoints analysis, Awadh et al. [10] suggested that the association between ZIKV and microcephaly is causal. But they also pointed out the need for further studies using animal models that will be helpful in concluding this assessment. Other researchers had also concluded that although there is substantial evidence suggesting a causal link between ZIKV and microcephaly, further studies are warranted to solidify this association [11]. In an in vitro study, McGrath et al. [12] demonstrated that human neural stem cells, from which the brain is formed, are highly susceptible to infection with ZIKV, possibly the cause of ZIKV-associated neuropathology during development. Emerging evidence also hints at an association of ZIKV infection with other birth defects [13]. In this context, it becomes pertinent to understand the natural transmission and pathogenesis of ZIKV infection by undertaking in vivo studies.

In earlier studies, the intracerebral inoculation route was used to study ZIKV pathogenesis, but this is not the natural route of ZIKV infection [14]. To mimic the natural mode of transmission of ZIKV by A. aegypti, suitable animal models are required [15]. In this study, we allowed infant CD1 mice to be fed upon by ZIKV-infected $A$. aegypti mosquitoes, thus mimicking the natural route of ZIKV transmission. Real-time RT-PCR, immunohistochemistry (IHC), and histopathological studies were performed to observe the site of predilection for ZIKV multiplication and disease development thereafter.

\section{Materials and Methods}

Mice and Ethics Statement

Two strains of mice, namely BALB/c and CD1, were used for this study. Adult BALB/c mice (aged 6-8 weeks) and infant CD1 mice ( 1 day old) were obtained from Animal House, National Institute of Virology, Pune. All the experiments carried out during this study were approved by the Institutional Animal Ethics (CPCSEA registration No. 43/GO/C/1999/CPCSEA) and Biosafety Committees.

\section{ZIKV Propagation in Cell Culture and Infant Mice}

The MR-766 strain of ZIKV, grown in C6/36 cells with 5 passages and a TCID50 titer of $10^{3.82} \mathrm{TCID}_{50} / \mathrm{mL}$ was received from the European Union and European Virus Archive goes Global project. The available Gene Bank sequence ID for this strain is DQ859059. T-25 cm $\mathrm{cm}^{2}$ tissue culture flasks of Vero CCL-81 cells with nearly $80 \%$ confluency were infected at a multiplicity of infection of 0.01 to prepare stock virus suspension. The cells were incubated at $37^{\circ} \mathrm{C}$ with $5 \% \mathrm{CO}_{2}$. Three passages were performed to raise the titer, with daily observation for any cytopathic effect (CPE). Supernatant from the culture flasks was then harvested and stored at $-80^{\circ} \mathrm{C}$ till further use. ZIKV was also titrated in Vero CCL-81 cells using standard protocol [16].

Control cells as well as ZIKV-infected Vero CCL-81 cells were also subjected to immunofluorescence assay (IFA). Checkerboard titration IFA of different dilutions of ZIKV hyperimmune serum raised in mice against dilutions of FITC-conjugated goat antimouse IgG (Sigma, USA) was performed and screened for optimum fluorescence under a compound fluorescent microscope (Olympus, BX61, USA).

ZIKV stock was prepared by intracerebral inoculation of the abovementioned virus (from the Vero CCL-81 cell culture) in the infant CD1 mice. After euthanizing, 10\% (weight/volume) mouse brain suspension was prepared in PBS (1.25\% BSA). This suspension was used for raising the hyperimmune serum against ZIKV, which was used later as a primary antibody for IHC.

\section{Raising Hyperimmune Serum against ZIKV in Mice for IFA} and IHC

For raising hyperimmune sera against ZIKV, a total of 15 $\mathrm{BALB} / \mathrm{c}$ adult mice (aged 6-8 weeks) were used. Prebleeding was performed and 100-200 $\mu \mathrm{L}$ of blood was collected in Eppendorf tubes and kept at room temperature for about $45 \mathrm{~min}$ to isolate the serum. The serum was separated by centrifuging at $4,000 \mathrm{rpm}$ for $15 \mathrm{~min}$ at $4{ }^{\circ} \mathrm{C}$. ZIKV immunization in mice was performed using infected infant CD1 mouse brain suspension (from the 2nd passage). This was mixed in equal volumes with Freund's complete adjuvant, and emulsified by pipetting for 10 min using an 18-gauge needle attached to a $2-\mathrm{mL}$ syringe; $100 \mu \mathrm{L}$ of this emulsion was injected into each mouse subcutaneously. The mice were monitored twice daily for any clinical signs. Three more booster doses were given at 14-day intervals, and Freund's incomplete adjuvant was used. Final bleeding was performed at the end of the 14th day after the 3rd booster, and the mice were euthanized with the help of a $\mathrm{CO}_{2}$ chamber. The serum was separated as described earlier. The euthanized mice were disposed of as per the standard biowaste disposal protocol of our facility approved by the Institutional Biosafety Committee.

Real-Time RT-PCR Analysis of Viral RNA

For confirmation of ZIKV in the organs of the infected mice, mosquitoes and tissue culture fluid real-time RT-PCR of Zika viral RNA was performed using primers and the probe set ZIKV 1086, ZIKV 1162c, and ZIKV 1107-FAM as described by Lanciotti et al. 
[17]. Briefly, RNA was extracted from $140 \mu \mathrm{L}$ of infected culture supernatant/mosquito pool/tissue suspension of different organs using the QIAamp viral RNA mini kit (Qiagen, Germany) as per the manufacturer's protocol. The extracted RNA was finally eluted in $50 \mu \mathrm{L}$ of nuclease-free water and stored at $-80^{\circ} \mathrm{C}$ till further use. Real-time RT-PCR was performed using SuperScript III One Step RT-PCR kit as described by Lanciotti et al. [17] under the following conditions: $50^{\circ} \mathrm{C}$ for $30 \mathrm{~min}, 95^{\circ} \mathrm{C}$ for $2 \mathrm{~min}$ followed by 40 cycles of $95^{\circ} \mathrm{C}$ for $15 \mathrm{~s}$, and $60^{\circ} \mathrm{C}$ for $1 \mathrm{~min}$. Positive results were interpreted for reactions which generated a fluorescence curve that crossed the threshold within $<38$ cycles. Negative control mice organs/mosquito negative controls/control tissue culture fluid were treated similarly and used during the assay.

Infection of Female Mosquitoes with ZIKV by the Oral Route and Confirmation of Virus Multiplication by Real-Time $R T-P C R$

Female A. aegypti mosquitoes were obtained from the laboratory colony maintained at the National Institute of Virology, Pune. Approximately 4-5 days after emerging, they were infected with ZIKV by allowing them to feed on an infectious blood meal as described earlier $[18,19]$. The infected mosquitoes were housed in cylindrical plastic jars ( $3 \mathrm{~cm}$ in diameter and $7 \mathrm{~cm}$ in height) covered with mosquito netting. These jars were kept in a wire-mesh cage $(30 \times 30 \times 38 \mathrm{~cm})$, which was covered with a wet lint cloth to provide the required temperature of $28 \pm 2{ }^{\circ} \mathrm{C}$ and $85 \pm 5 \%$ relative humidity, and kept in a biological oxygen demand incubator. During the entire period of incubation, the mosquitoes were maintained on $10 \%$ glucose solution soaked in cotton wool. For confirmation of ZIKV propagation, 5 mosquitoes were harvested on the 6th postinoculation day (PID), triturated in $1 \mathrm{~mL}$ of sterile $1 \mathrm{X}$ PBS, and centrifuged at $10,000 \mathrm{rpm}$ for $30 \mathrm{~min} ; 140 \mu \mathrm{L}$ of the supernatant was used for RNA extraction and $5 \mu \mathrm{L}$ of RNA was used in real-time RT-PCR for the detection of Zika viral RNA as described above.

\section{ZIKV Transmission by Infected Mosquitoes to Infant Mice}

As described above, after being infected with ZIKV by the oral route, the mosquitoes were allowed to feed on 2-day old infant CD1 mice $(n=8)$ on the 7th PID, as described earlier by Banerjee et al. [20]. For 4-5 days, the mice were monitored twice daily for the appearance of sickness and clinical symptoms. Brain, liver, spleen, kidneys, intestines, heart, and lungs were harvested. Blood and urine were collected prior to euthanization. The harvested organs as well as the serum and urine samples were tested for the presence of ZIKV by real-time RT-PCR as described above. Harvested organs were also subjected to histopathology and IHC as per the procedure given by Aliota et al. [21].

\section{Histopathology and IHC Analysis of ZIKV-Infected Mouse} Organs

The organs from experimentally infected mice $(n=7)$ were fixed for about $74 \mathrm{~h}$ in $10 \%$ neutral-buffered formalin. Paraffinembedded organs were sectioned (thickness, 4-5 $\mu \mathrm{m}$ ) and stained with hematoxylin and eosin (H\&E) stain. The control samples were processed simultaneously and similarly. Slides were observed under a light microscope.

For IHC, sections were fixed over poly-L-lysine-coated slides and processed as per the manufacturers' instructions (Dako, Denmark). Polyclonal antibody (diluted at 1:1,600) generated in the
Table 1. Replication confirmation of Zika virus in Indian A. aegypti mosquitoes

\begin{tabular}{llll}
\hline \multicolumn{5}{c}{ Membrane-fed mosquitoes } \\
\cline { 2 - 4 } & Ct value & $\log$ of copy number & \\
& & $\begin{array}{l}\text { copy number/ } \\
5 \mu \mathrm{L} \text { of RNA }\end{array}$ \\
\hline Day 0 & 28.4 & 6.6417 & $4.3 \times 10^{6}$ \\
PID 6 & 18.36 & 10.0610 & $1.1 \times 10^{10}$ \\
\hline
\end{tabular}

Five mosquitoes were subjected to real-time RT-PCR at day 0 and on the 6th postinfection day (PID).

${ }^{a}$ From the standard curve.

$\mathrm{BALB} / \mathrm{c}$ mice against ZIKV was used as primary antibody and Envision FLEX/HRP-labeled IgG was used as secondary antibody. The slides were stained with substrate $3-3^{\prime}$ diaminobenzidine tetra hydrochloride and $\mathrm{H}_{2} \mathrm{O}_{2}$ solution as per the manufacturer's instructions for $2 \mathrm{~min}$ at room temperature. For the negative control, the control sections were run in parallel. After IHC staining, sections were counterstained with $\mathrm{H} \& \mathrm{E}$ and examined by light microscopy.

\section{Results}

The CPE Was Prominent in ZIKV-Infected Vero Cells

In Vero CCL-81 cells infected with ZIKV, a CPE was observed on the 3rd PID, and comprised morphological changes like the rounding of cells and cell death. After the 3rd PID, cell detachment was observed (Fig. 1). During the in vitro virus titration experiment, $\mathrm{CPE}$ was observed in all the wells of Vero CCL-81 cells infected with ZIKV, ranging from $10^{-1}$ to $10^{-4}$ dilutions. The Reed-Muench method showed approximately $10^{5.5} \mathrm{TCID}_{50} / \mathrm{mL}$. The control wells did not show any CPE until the 7th PID.

The IFA results showed that ZIKV hyperimmune sera raised in mice at 1:50 dilution and 1:100 dilution of FITCconjugated goat anti-mouse IgG (Sigma, USA) produced optimum fluorescence for the detection of ZIKV in infected Vero CCL-81 cells (Fig. 1).

\section{Confirmation of ZIKV Multiplication in Infected}

Mosquitoes on the 6th PID by Real-Time RT-PCR

The presence of Zika viral RNA was observed in mosquitoes infected on day 0 and on the 6th PID, and was assessed by Zika-specific real-time RT-PCR. Confirmation of ZIKV replication in the infected Indian strain of A. aegypti mosquitoes on the 6th PID (Ct value: 18.3 vs. 28.4 at day 0 ) showed that ZIKV was replicating efficiently in these mosquitoes (Table 1). 


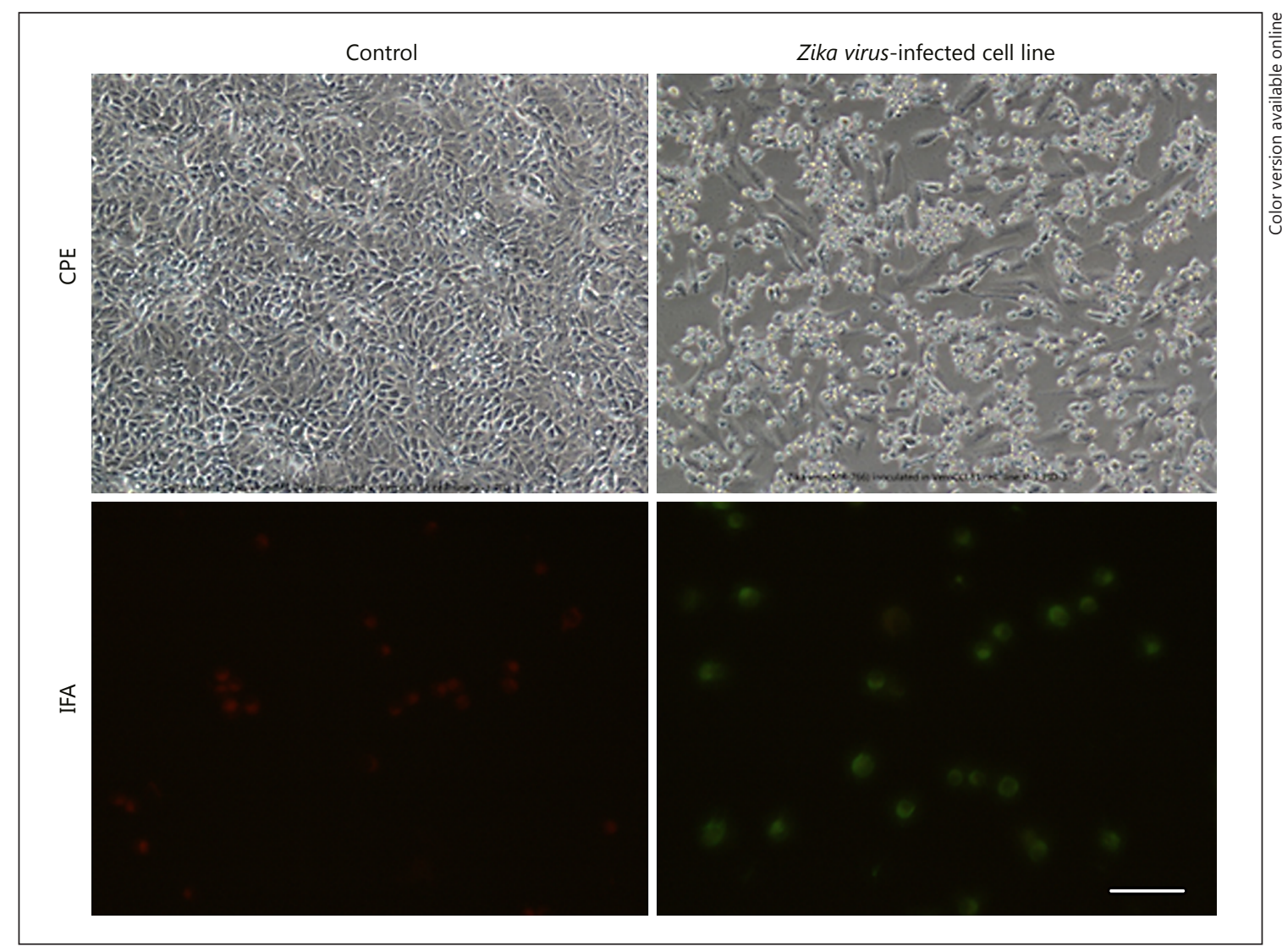

Fig. 1. Cytopathic changes as observed by bright-field and fluorescence microscopy in controls and the Zika virusinfected Vero CCL-81 cell line. IFA, immunofluorescence assay; CPE, cytopathic effect. Scale bar, $100 \mu \mathrm{m}$.

Observed Clinical Signs in ZIKV-Infected Mice and the Presence of Zika Viral RNA in Infected Mice ZIKV-infected mosquitoes were allowed to feed on infant CD1 mice. Mice showed sickness around the 4 th $/ 5$ th PID. Symptoms included trembling, solitary behavior, nervous signs, no movement, and lethargy. In the terminal stages, mice became moribund. The harvested organs from the euthanized mice, i.e., the brain, liver, spleen, kidneys, intestines, heart, and lungs, and the blood and urine samples were tested for Zika viral RNA. Zika viral RNA was mostly detected in the brain (Ct value: 11 ) versus the other organs and tissues (blood [Ct value: 34], heart [Ct value: 28], kidneys [Ct value: 29], lungs [Ct value: 30]), intestines [Ct value: 26], liver/spleen [Ct value: 34], and urine [Ct value: 32]).

From this experiment, it was demonstrated that although the virus had disseminated and multiplied in all the organs of the mice after the bite by the infected mosquitoes, the highest viral load was present in the brain, indicating that ZIKV shows tropism for this tissue.

\section{Histopathological and IHC Analysis Showed}

Numerous Abnormalities in Mice Infected with ZIKV

The detailed histopathological examination of the brains of infected mice $(n=7)$ showed typical features of neuronal degeneration with vacuolar changes in the cerebrum, neuropil, and hippocampus region. Neuronal necrosis with hypereosinophilic cytoplasm, nuclear fragmentation, and pyknosis were evident in multiple foci of the brain (Fig. 2a-d). The increased cellular population of microglia as well as polymorphonuclear cells and lymphocytes at the foci of neuronal degeneration were observed along with satellitosis. Perivascular cuffing of inflammatory cells was also evident, suggestive of a reactive inflammatory response at the vascular endothelium.

Kidneys from the infected mice (in 4 of 7) revealed mild degenerative pathological changes including cellular swelling and karyomegaly of the tubular epithelium, focal areas of tubular vacuolation, and infiltration of polymorphonuclear cells in the renal parenchyma. The lungs (in 4 of 7 ) also showed focal and minimal but defi- 

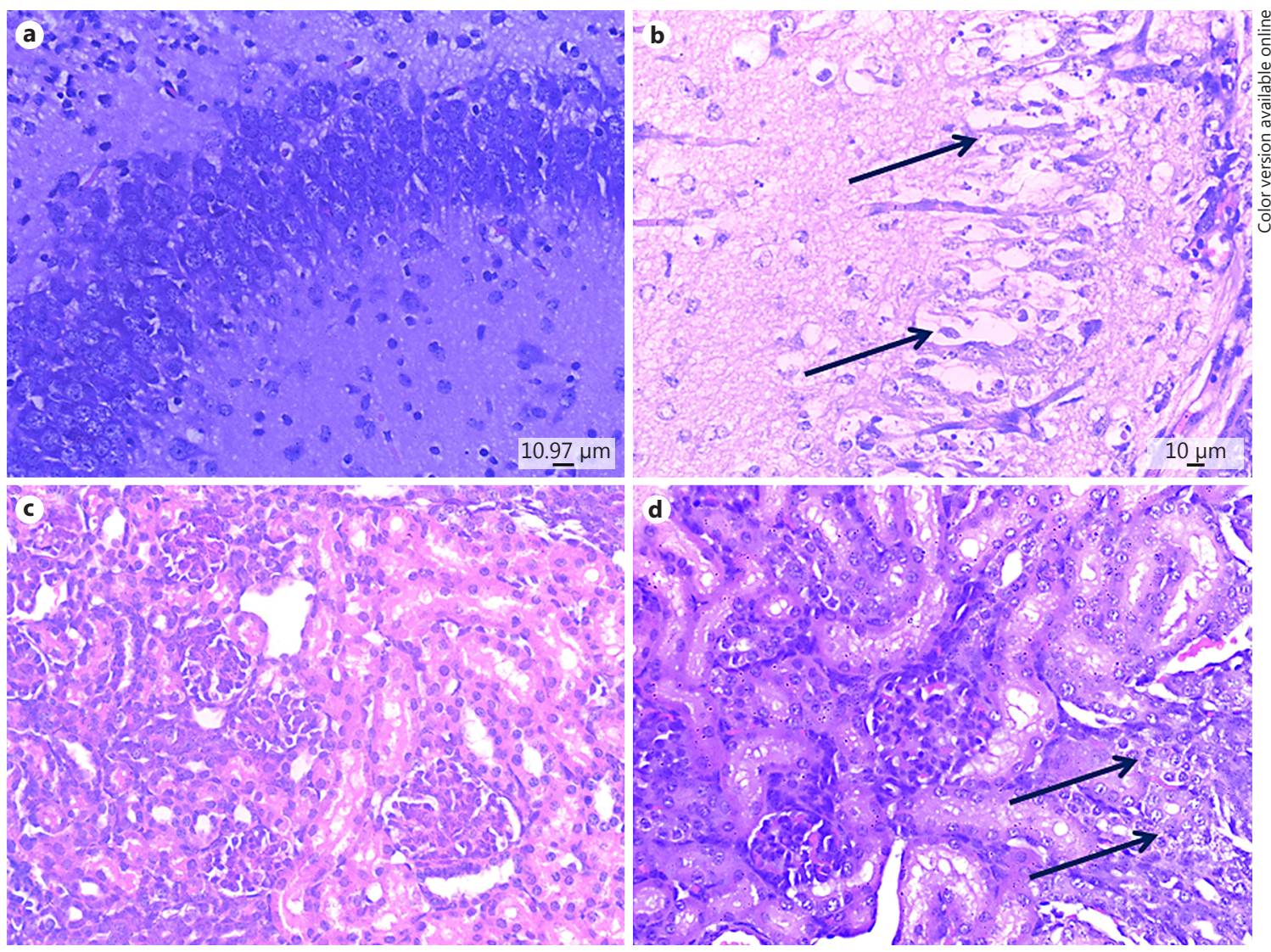

Fig. 2. Histopathological changes in various organs of infant CD1 mice after being bitten by ZIKV-infected mosquitoes (arrows). $\times 400$. a A control brain depicting normal neuronal tissue. $\mathbf{b}$ Brain of an infected mouse depicting degeneration and neuronal necrosis. c A control kidney. d Kidney of an infected mouse depicting degenerative pathological changes.

nite pathological lesions, comprising focal emphysematous lesions and consolidation of alveolar parenchyma with the presence of few mononuclear cells. The liver tissue sections from 2 infected mice showed focal degenerative changes in the hepatocytes, with granular and vacuolar cytoplasmic features and the occasional loss of the nucleus. In addition, diffused foci of extramedullary hematopoiesis with few megakaryocytes in the hepatic parenchyma were also observed. The heart tissue sections from the infected group showed focal congestion in the coronary blood vessels.

The histological examination of organs from the control group $(n=6)$ showed normal histomorphology with changes indicative of the early stage of infant development like extramedullary hematopoiesis.

\section{IHC Observations}

The IHC of brain tissue sections from the infected mice showed localization of the viral antigen, principally in the neuronal body and cytoplasm. The neuronal tissue from the cerebral cortical region exhibited a higher accumulation of viral antigen than in other regions of brain. The hippocampus also revealed the presence of viral antigen in the neurons. Renal sections also showed more marked localization of viral antigen in the tubular epithelium than in the glomeruli. Diffuse accumulation of viral antigen in the renal tubules was a prominent observation (Fig. 3a-d).

\section{Discussion}

Data suggested that CD1 mice should be explored as a suitable model for understanding ZIKV natural transmission and pathogenesis. We chose infant CD1 mice for 2 reasons; firstly, they will be immunocompromised, and secondly, they will be easily accessible to mosquitoes for biting due to the absence of hair on their bodies. Within 4-5 days of being bitten by the mosquitoes, the mice fell 

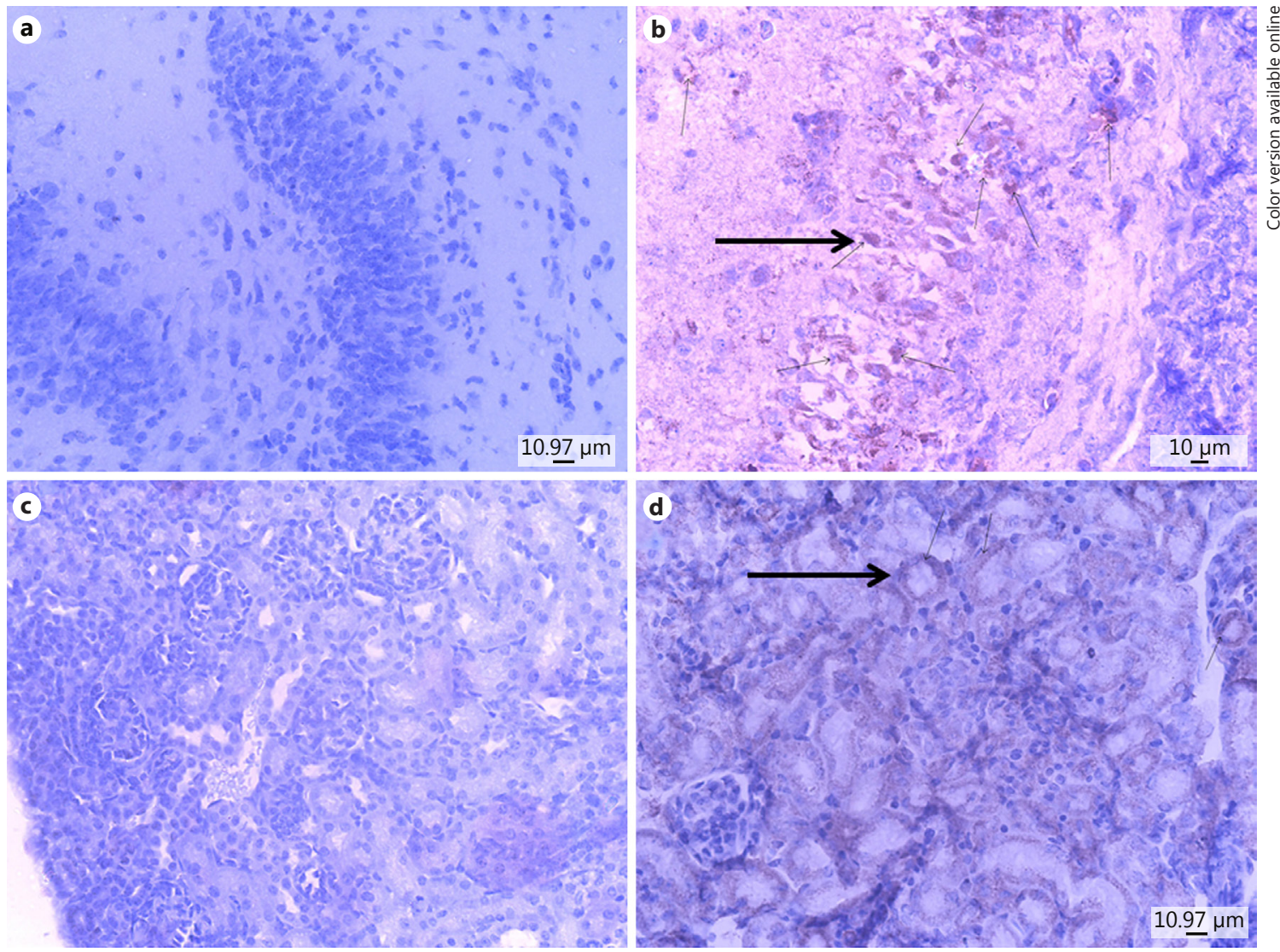

Fig. 3. Immunohistochemical changes in the organs of infant CD1 mice after being bitten by infected mosquitoes (arrows). $\times 400$. a A control brain depicting normal neuronal tissue. $\mathbf{b}$ Brain of an infected mouse depicting the presence of the viral antigen in the neurons, with degeneration and neuronal necrosis. c A control kidney. $\mathbf{d}$ Kidney of an infected mouse depicting the presence of the viral antigen.

sick, showing that they were highly susceptible to challenge by ZIKV.

This kind of exploratory study has been conducted by many researchers. In earlier studies, adult mouse models were used to study ZIKV pathogenesis. Dowall et al. [22] used IFN- $\alpha / \beta$ receptor-deficient (A129) mice, and found them to be highly susceptible to ZIKV infection. Aliota et al. [21] used IFN- $\alpha / \beta$ - and $\gamma$-deficient (AG129) mice, and concluded the same. In both of these studies, the adult mice used were knocked out for IFN genes. In our study, though there was routine use of the mice, the ZIKV infection was still perpetuated in these animals, highlighting the fact that infant CD1 mice are highly susceptible to ZIKV infection. Miner et al. [23] developed an adult pregnant mouse model (Ifnar1-/-), in which maternal inoculation with ZIKV resulted in fetal demise with lesions in the fetal brain, showing transplacental transmission of the virus and its tropism for the brain.
The ZIKV positivity in mouse organs leading to disease and eventually death demonstrated that infant CD1 mice are highly susceptible to natural ZIKV infection and could therefore be used for future studies on ZIKV. We also showed that infected $A$. aegypti mosquitoes can transmit the infection effectively to suckling mice. These mosquitoes harbor this virus in their salivary glands, which explains its easy transmission when feeding on suckling mice [24]. Zika viral RNA was detected in different visceral organs including the brain, kidneys, liver, lungs, etc. ZIKV-infected mice also showed extensive tissue degeneration in the brain and kidneys, which was probably the major cause of death.

To confirm the predilection site of ZIKV, we assessed Zika viral RNA levels in different tissues by real-time RTPCR. In this particular experiment, we did a qualitative assessment. The virus was detected in the blood, brain, liver, spleen, kidneys, intestines, lungs, heart, and urine of 
the infected infant mice, but the viral load, determined by the Ct values, was higher in the brain tissue than in the other organs. Earlier studies had similar results with other strains of mice [21-23].

The histopathological analysis also revealed the presence of numerous microscopic lesions characteristic of ZIKV infection, primarily in the peripheral regions of the brain. The overall picture of the sections of the brain in the infected group suggested diffused neuropathy (degeneration of neurons and neuronal loss by necrosis) along with inflammatory changes (gliosis and satellitosis). Such lesions were also predominant in the hippocampus region. It is quite interesting that similar lesions were observed previously when mice were inoculated intracerebrally [23]. Lesions were also noticed in the lungs, liver, heart, and kidneys, which could be a direct response to the viral infection or a reactive process. Cases of ZIKV infection have been associated with microcephaly, calcifications in the brain, and other abnormalities [13], but no such lesions were observed in our study. This may have been due to the fact that the development of these lesions takes time, while we observed only the acute phase of the infection. Nevertheless, the predilection of this virus for nervous tissue, along with the extensive pathological changes it caused in the brain, shows that the association of ZIKV infection and microcephaly and other neuronal malformations is likely a direct example of a cause-and-effect relationship.

To confirm whether the lesions were a direct effect of the viral infection, IHC was performed. This confirmed the histopathological results, shown by the maximum staining of the ZIKV antigen in the brain of the infected mice including the cortical and hippocampus regions. We can assume that ZIKV has an affinity for these regions among other regions of brain, e.g., the vascular endothelium (not detected). Interestingly, apart from in the brain, ZIKV antigen could only be detected in the renal glomeruli. Martines et al. [25], while examining newborn human cases of microcephaly, could also not detect ZIKV antigen outside the brain. Our results and those of earlier studies confirm the preponderance of ZIKV for the brain, and testify to its association with neurological malformations in affected cases. We firmly believe that the infant murine model developed by this work will pave the way for more advanced studies aimed at understanding ZIKV vertical transmission and determining strain pathogenicity, eventually advancing our knowledge of ZIKV.

To summarize, ZIKV is an evolving emerging disease and a global threat, unless it can be contained effectively and efficiently, especially in areas where its vector is in abundance including India. Migration patterns of ZIKV to different geographical regions outside Africa and its association with deaths and neurological manifestations in children born with the disease are a matter of significant concern. A pertinent need has arisen to explore possibilities for the containment of ZIKV. These, in turn, demand advanced knowledge regarding its pathogenesis and natural replication cycle. Through this work, we have demonstrated a natural method for infecting infant CD1 mice with ZIKV. Our murine model can also be used to test the efficacy of vaccines and therapeutics, and for epidemiological surveillance in high-risk regions.

\section{Acknowledgement}

We thank Dr. Soumya Swaminathan, Secretary, Department of Health Research, and the Director General, ICMR, New Delhi, India, for the intramural fund support and keen interest in this proactive public health research. The authors express sincere thanks to Mr. Prasad Sarkale, Mr. Rajen Lakra, Ms. Savita Patil, Mr. Manoj Kadam, and Mr. Kumar Baghmare for technical support during this study.

\section{Disclosure Statement}

The authors declare no conflicts of interest.

\section{Funding Sources}

We received a grant from the Indian Council of Medical Research, New Delhi, India. The funders had no role in study design, data collection and interpretation, or the decision to submit the work for publication.

References

1 Dick GW, Kitchen SF, Haddow AJ: Zika virus. I. Isolations and serological specificity. Trans R Soc Trop Med Hyg 1952;46:509-520.

2 Lazear HM, Diamond MS: Zika virus: new clinical syndromes and its emergence in the Western hemisphere. J Virol 2016;90:48644875.

-3 Fauci AS, Morens DM: Zika virus in the Americas - yet another arbovirus threat. N Engl J Med 2016;374:601-604.

4 Zanluca C, de Melo VCA, Mosimann ALP, dos Santos GIV, dos Santos CND, Luz K: First report of autochthonous transmission of $\mathrm{Zika}$ virus in Brazil. Mem Inst Oswaldo Cruz 2015; 110:569-572.

5 McCrae AW, Kirya BG: Yellow fever and Zika virus epizootics and enzootics in Uganda. Trans R Soc Trop Med Hyg 1982;76:552-562. 
-6 Marchette NJ, Garcia R, Rudnick A: Isolation of Zika virus from Aedes aegypti mosquitoes in Malaysia. Am J Trop Med Hyg 1969;18: 411-415.

$>7$ Messina JP, Kraemer MU, Brady OJ, Pigott DM, Shearer FM, Weiss DJ, Golding N, Ruktanonchai CW, Gething PW, Cohn E, Brownstein JS, Khan K, Tatem AJ, Jaenisch T, Murray CJ, Marinho F, Scott TW, Hay SI: Mapping global environmental suitability for Zika virus. eLife 2016;5:e15272.

-8 Musso D, Cao-Lormeau V M, Gubler DJ: Zika virus: following the path of dengue and chikungunya? Lancet Lond Engl 2015;386:243244.

>9 Dyer O: Zika virus spreads across Americas as concerns mount over birth defects. BMJ 2015; 351:h6983.

10 Awadh A, Chughtai AA, Dyda A, Sheikh M, Heslop DJ, MacIntyre CR: Does Zika virus cause microcephaly - applying the Bradford Hill viewpoints. PLoS Curr 2017, DOI: $10.1371 /$ currents.outbreaks.2fced6e886074f6 db162a00d4940133b.

11 Wang JN, Ling F: Zika virus infection and microcephaly: evidence for a causal link. Int J Environ Res Public Health 2016;13.

-12 McGrath EL, Rossi SL, Gao J, Widen SG, Grant AC, Dunn TJ: Differential responses of human fetal brain neural stem cells to Zika virus infection. Stem Cell Reports 2017;8: 715-727.
13 Mlakar J, Korva M, Tul N, Popović M, Poljšak-Prijatelj M, Mraz J, Kolenc M, Rus KR, Vipotnik TV, Vodusek VF, Vizjak A, Pizem J, Petrovec M, Zupanc TA: Zika virus associated with microcephaly. N Engl J Med 2016;374:951-958.

14 Fagbami AH: Zika virus infections in Nigeria: virological and sero-epidemiological investigations in Oyo State. J Hyg 1979;83:213-219.

15 Tetro JA: Zika and microcephaly: causation, correlation, or coincidence? Microbes Infect 2016;18:167-168.

16 Reed LJ, Muench H: A simple method of estimating fifty per cent endpoints. Am J Hyg 1938;27:493-497.

17 Lanciotti RS, Kosoy OL, Laven JJ, Velez JO, Lambert AJ, Johnson AJ, Stanfield SM, Duffy MR: Genetic and serologic properties of Zika virus associated with an epidemic, Yap State, Micronesia, 2007. Emerg Infect Dis 2008; 14: 1232-1239.

18 Harada M, Matsuoka H, Suguri S: A convenient mosquito membrane feeding method. Med Entomol Zool 1996;47:103-105.

19 Mourya D, Gokhale MD, Barde PV, Padbidri VS: A simple artificial membrane-feeding method for mosquitoes. Trans Royal Soc Trop Med 2000;94:460.
20 Banerjee K, Mourya DT, Malunjkar AS: Susceptibility and transmissibility of different geographical strains of Aedes aegypti mosquitoes to Chikungunya virus. Indian J Med Res 1988;87:134-138.

21 Aliota MT, Caine EA, Walker EC, Larkin KE, Camacho E, Osorio JE: Characterization of lethal Zika virus infection in AG129 mice. PLoS Negl Trop Dis 2016;10:e0004750.

22 Dowall, SD, Graham VA, Rayner E, Atkinson B, Hall G, Watson R, Bosworth A, Bonney L, Kitchen S, Hewson R: A susceptible mouse model for Zika virus infection. PLoS Negl Trop Dis 2016;10:e0004658.

23 Miner J, Cao B, Govero J, Noguchi KK, Mysorekar IU, Diamond MS: Zika virus infection during pregnancy in mice causes placental damage and fetal demise. Cell 2016:165:10811091.

24 Musso D, Gubler DJ: Zika virus. Clin Microbiol Rev 2016:29:487-524.

25 Martines RB, Bhatnagar J, de Oliveira Ramos AM, Davi HPF, Iglezias SA, Kanamura CT, Keating MK, Hale G, Flannery LS, Muehlenbachs A, Ritter J, Gary J, Rollin D, Goldsmith CS, Steiner SR, Ermias Y, Suzuki T, Luz KG: Pathology of congenital Zika syndrome in Brazil: a case series. Lancet 2016;6736:3088330882. 